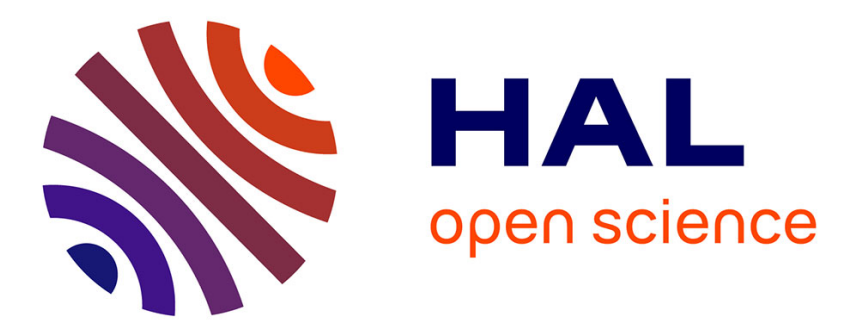

\title{
Toward the coupling of a discontinuous Galerkin method with a MoM for analysis of susceptibility of planar circuits
}

Caroline Girard, Stéphane Lanteri, Ronan Perrussel, Nathalie Raveu

\section{- To cite this version:}

Caroline Girard, Stéphane Lanteri, Ronan Perrussel, Nathalie Raveu. Toward the coupling of a discontinuous Galerkin method with a MoM for analysis of susceptibility of planar circuits. IEEE Transactions on Magnetics, 2014, 50 (2), pp.509-512. 10.1109/TMAG.2013.2282462 . hal-00958274

\section{HAL Id: hal-00958274 https://hal.science/hal-00958274}

Submitted on 12 Mar 2014

HAL is a multi-disciplinary open access archive for the deposit and dissemination of scientific research documents, whether they are published or not. The documents may come from teaching and research institutions in France or abroad, or from public or private research centers.
L'archive ouverte pluridisciplinaire HAL, est destinée au dépôt et à la diffusion de documents scientifiques de niveau recherche, publiés ou non, émanant des établissements d'enseignement et de recherche français ou étrangers, des laboratoires publics ou privés. 


\title{
Towards the coupling of a discontinuous Galerkin method with a MoM for analysis of susceptibility of planar circuits
}

\author{
Caroline Girard ${ }^{1,2}$, Stéphane Lanteri ${ }^{2}$, Ronan Perrussel ${ }^{1}$, and Nathalie Raveu ${ }^{1}$ \\ ${ }^{1}$ Université de Toulouse ; INPT, UPS ; CNRS ; LAPLACE ; ENSEEIHT, Toulouse, France \\ ${ }^{2}$ NACHOS project-team, INRIA Sophia Antipolis-Méditerranée, Sophia Antipolis, France
}

\begin{abstract}
We aim at coupling a method of moments, the Wave Concept Iterative Procedure, and the Hybridizable Discontinuous Galerkin method to study electromagnetic susceptibility of innovative planar circuits in 3D. Hybridizing the Wave Concept Iterative Procedure with volumic methods like the Frequency Domain Transmission Line Matrix method, the Finite Element Method and the Hybridizable Discontinuous Galerkin method in 2D is a first step for the validation of the proposed coupling technique. The considered problem is Maxwell's equations in the frequency domain. Three test cases in 2D and a preliminary result in 3D are provided.
\end{abstract}

Index Terms-Microwave propagation, method of moments, finite element methods.

\section{INTRODUCTION}

$\mathbf{T}$ HE Wave Concept Iterative Procedure (WCIP) [1] is a method adapted to the study of microwave circuits, solving Maxwell's equations in guided and stratified structures. Nevertheless, it cannot characterize circuits with dielectric inhomogeneities [2]. This has naturally led to the issue of hybridization of the WCIP with volumic methods such as the finite element method (FEM), the hybridized discontinuous Galerkin method (HDG) [3] or a method based on transmission line theory, the Frequency Domain Transmission Line Matrix method (FDTLM). A hybridization with the FDTLM has already been achieved in [4]. The advantage of the HDG method lies in its flexibility with regards to the type of mesh used for the discretization of the volumic part (it can be unstructured, hybrid, non-conforming) and in its adaptivity in the polynomial approximation order; this interpolation order can be chosen lower near the interface (if the field is not regular because of discontinuities) and higher some elements further.

This work is concerned with circuit modeling in the high frequency range. It describes the hybridization of numerical methods in the frequency domain to study the electromagnetic susceptibility of planar circuits. We aim at detecting potential perturbations induced in a circuit by an external electromagnetic source. In this purpose, the planar circuit is illuminated by a wave and we calculate the electric field and current induced on the considered circuit. Three 2D [5] and one $3 \mathrm{D}$ test problems are dealt with in this paper to validate the resulting hybrid methods. TM and TE cases have been studied, but only TE results are presented here (conclusions for the TM case being similar). The ultimate goal is to be able to treat more complex 3D configurations.

\section{HYBRIDIZATION PRINCIPLE}

For the sake of simplicity, the computational domain is decomposed into two subdomains as shown in Fig. 1. Boundaries

Manuscript received June 28, 2013; revised 2013. Corresponding author: C. Girard (email: Caroline.Girard@inria.fr).

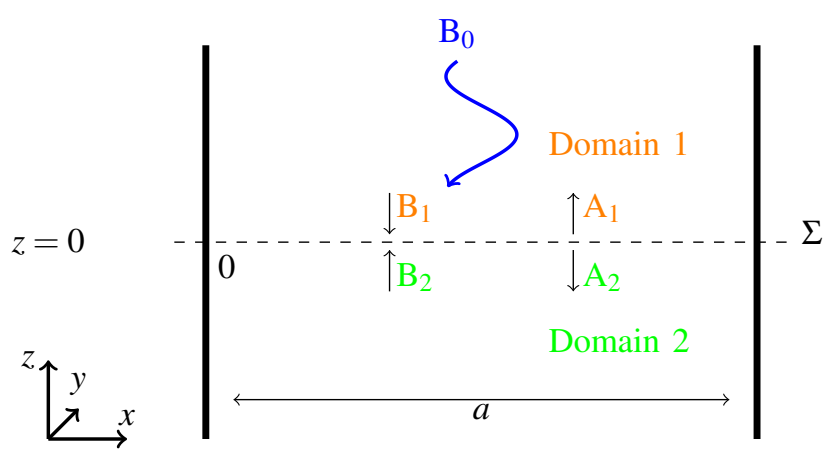

Fig. 1. Case under study, separation between both domains 1 and 2 according to the interface $\Sigma$.

at $x=0$ and $x=a$ are metallic walls. In the hybridization context, the wave propagation in domain 1 is numerically modeled by the WCIP whereas in domain 2 it is addressed with a volumic method; the connection is achieved at the interface $\Sigma$. The WCIP domain is not bounded, whereas the other domain is bounded by a metallic wall. We describe below the iterative process of the WCIP and then proceed to the formulation of the linear system characterizing the hybridization approach. The WCIP is based on outgoing waves $\mathbf{A}_{\mathbf{1}}$ and $\mathbf{A}_{\mathbf{2}}$ and incoming waves $\mathbf{B}_{\mathbf{1}}$ and $\mathbf{B}_{\mathbf{2}}$ on $\Sigma$ (see Fig. 1). The iterative process without coupling writes

$$
\left\{\begin{array}{l}
\mathbf{B}_{1}^{(k+1)}=\mathrm{FMT}^{-1} \Gamma_{1} \mathrm{FMT}_{1}^{(k)}+\mathbf{B}_{0}, \\
\mathbf{B}_{2}^{(k+1)}=\mathrm{FMT}^{-1} \Gamma_{2} \text { FMT }_{2}^{(k)},
\end{array}\right.
$$

with vectors $\mathbf{A}_{i}^{(k)}, \mathbf{B}_{i}^{(k)}$ containing the discrete representations of the waves $\mathbf{A}_{i}, \mathbf{B}_{i}$ at the iteration $k, \Gamma_{i}$ the diagonal matrix composed of modal diffraction coefficients $\Gamma_{i, n}$, FMT standing for Fast Modal Transform [1], and $\mathbf{B}_{0}$ representing the source excitation. In the TE case, $\Gamma_{1, n}$ is given by

$$
\Gamma_{1, n}^{\mathrm{TE}}=\frac{1-Z_{0} Y_{1, n}^{\mathrm{TE}}}{1+Z_{0} Y_{1, n}^{\mathrm{TE}}} \text { with } Y_{1, n}^{\mathrm{TE}}=\frac{\sqrt{\left(\frac{n \pi}{a}\right)^{2}-k_{0}^{2}}}{j \omega \mu_{0}},
$$


where $Y_{1, n}^{\mathrm{TE}}$ corresponds to a mode of order $n$ admittance

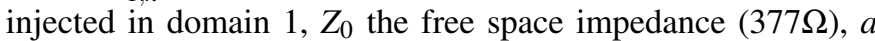
the distance between metallic slabs $(a=1.27 \mathrm{~cm}), k_{0}$ the wave number in vacuum at frequency $f_{0}=16 \mathrm{GHz}$, which gives $k_{0}=335 \mathrm{rad} / \mathrm{m}, \omega=10 \times 10^{10} \mathrm{rad} / \mathrm{s}$ and $\mu_{0}=1.26 \times 10^{-6} \mathrm{H} \cdot \mathrm{m}^{-1}$. The transmission operator $\mathrm{S}$ between both domains satisfies

$$
\left(\begin{array}{c}
\mathbf{A}_{1}^{(k)} \\
\mathbf{A}_{2}^{(k)}
\end{array}\right)=\mathrm{S}\left(\begin{array}{c}
\mathbf{B}_{1}^{(k)} \\
\mathbf{B}_{2}^{(k)}
\end{array}\right)=\left(\begin{array}{ll}
S_{11} & S_{12} \\
S_{21} & S_{22}
\end{array}\right)\left(\begin{array}{l}
\mathbf{B}_{1}^{(k)} \\
\mathbf{B}_{2}^{(k)}
\end{array}\right),
$$

where $S_{11}$ and $S_{12}$ are $N$-sized matrices ( $N$ being the number of segments on $\Sigma$ ), respectively equal to -1 and 0 on metal segments and respectively equal to 0 and 1 on insulator segments. For instance, when there is no metal, $S_{11}$ is filled with zeros and $S_{12}$ is equal to the identity matrix.

Consequently, the linear system to be solved is

$$
\left(\mathrm{I}_{\mathrm{d}}-\left(\begin{array}{cc}
\mathrm{S}_{1}^{\mathrm{W}} & 0 \\
0 & \mathrm{~S}_{2}^{\mathrm{W}}
\end{array}\right) \mathrm{S}\right)\left(\begin{array}{l}
\mathbf{B}_{1} \\
\mathbf{B}_{2}
\end{array}\right)=\left(\begin{array}{c}
\mathbf{B}_{0} \\
0
\end{array}\right)
$$

where $I_{d}$ is the identity matrix, $S_{i}^{W}$ a matrix for the discretization by the WCIP, defined by

$$
\mathrm{S}_{\mathrm{i}}^{\mathrm{W}}=\mathrm{FMT}^{-1} \Gamma_{\mathrm{i}} \mathrm{FMT} .
$$

The coupling of the WCIP with a volumic method is obtained by replacing $S_{2}^{\mathrm{W}}$ by a matrix $S_{2}^{\mathrm{F}}$ for the discretization by a volumic method. Matrix $S_{2}^{F}$ has to characterize the relation between $\mathbf{B}_{2}$ and $\mathbf{A}_{2}$. In the hybridization setting, the wave $\mathbf{A}_{2}$ is introduced as a source term in the weak formulation. In 2D, TE and TM modes are uncoupled, which explains that waves are only along $y$-axis, according to Fig. 1 orientation. The corresponding weak formulation for the FEM is given by

$$
\begin{aligned}
& \int_{D_{2}} \nabla E_{y 2} \cdot \nabla w d s-k_{0}^{2} \int_{D_{2}} E_{y 2} w d s \\
& \quad+j k_{0} \int_{\Sigma} E_{y 2} w d l=2 j k_{0} \sqrt{Z_{0}} \int_{\Sigma} A_{y 2} w d l,
\end{aligned}
$$

where $D_{2}$ corresponds to domain $2, E_{y 2}$ is the electric field component along $y$-axis, $w$ stands for a test function and $A_{y 2}$ is the outgoing wave component along $y$-axis. The insertion of the source term for the HDG method is given by writing a conservativity condition [3] adding a specific term on $\Sigma$

$$
\begin{gathered}
\int_{\partial \mathcal{T}_{h}} \mathbf{n} \times\left(H_{x 2} \mathbf{x}+H_{z 2} \mathbf{z}\right) \bar{w} d l-\int_{\partial \mathcal{T}_{h}} \tau\left(E_{y 2}-\lambda_{h}\right) \bar{w} d l \\
+\int_{\Sigma} \frac{1}{Z_{0}} \lambda_{h} \bar{w} d l=\frac{2}{\sqrt{Z_{0}}} \int_{\Sigma} A_{y 2} \bar{w} d l
\end{gathered}
$$

where $\mathcal{T}_{h}$ is the triangulation of domain $2, \tau$ a stabilization parameter equal to $\frac{1}{Z_{0}}$ in our examples and $\lambda_{h}$ the hybrid variable introduced in HDG. It is a continuous variable defined at the interface of the elements, which represents the tangential electric field: $\lambda_{h}=\left.E_{y 2}\right|_{\mathcal{T}_{h}}$. In FEM, field $E_{y 2}$ is continuous at the frontiers between two elements whereas in HDG it is not. Indeed, fields $E_{y 2}, H_{x 2}$ and $H_{z 2}$ are calculated independently in each element and called, as a result, local fields. HDG solution is achieved calculating a hybrid variable $\lambda_{h}$ on all interfaces with (7) (after having eliminated local fields $E_{y 2}, H_{x 2}$ and $H_{z 2}$ with Maxwell's equations [3]) and then local fields are deduced. Incoming wave component $B_{y 2}$ can be calculated according to

$$
\begin{aligned}
B_{y 2} & =\frac{1}{2 \sqrt{Z_{0}}}\left(\left.E_{y 2}\right|_{\Sigma}-\left.\frac{Z_{0}}{j \omega \mu_{0}} \frac{\partial E_{y 2}}{\partial z}\right|_{\Sigma}\right), \\
\text { or } B_{y 2} & =\left.\frac{1}{\sqrt{Z_{0}}} E_{y 2}\right|_{\Sigma}-A_{y 2},
\end{aligned}
$$

due to the wave definitions (recalled in [4]). Equation (8) was mentioned in [6], but it appeared that equation (9) provided more accurate results and improved the convergence. $\left.E_{y 2}\right|_{\Sigma}$ was identified to variable $\lambda_{h}$ on $\Sigma$ to perform $B_{y 2}$ calculation. In the linear system (4), matrices are never explicitly built; (4) of size $2 N$ is solved by a restarted GMRES method [7]. Iterative solution is stopped when the norm of the residue has been divided by $10^{8}$. A comparison with the BICGSTAB method [8] is also discussed in the next section.

\section{NUMERICAL RESULTS}

Three test cases are considered in 2D: diffraction of a guided mode in vacuum on a perfect sheet (Fig. 2a), diffraction of a guided mode on a microstrip line in vacuum and diffraction of a guided mode on a microstrip line printed on an inhomogeneous substrate $\left(\varepsilon_{r, 1}=1\right.$ and $\varepsilon_{r, 2}=5$ ) (Fig. 2b). Some preliminary results are also given for a $3 \mathrm{D}$ configuration.

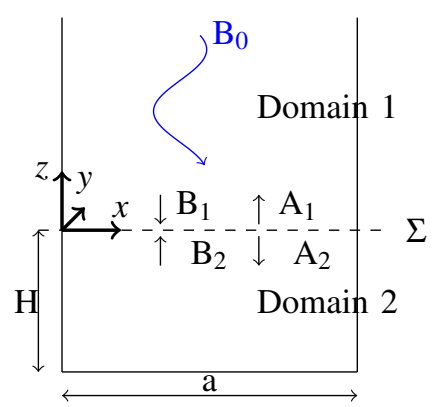

(a) Waveguide

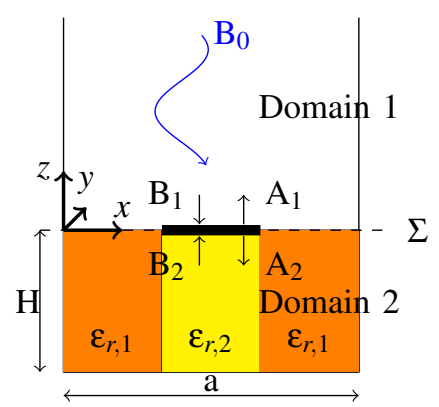

(b) Microstrip line
Fig. 2. Examples

\section{A. Diffraction of a guided mode on a perfect sheet}

The example of Fig. 2a is studied with $H=1.27 \mathrm{~cm}$ and $a=1.27 \mathrm{~cm}$ at $16 \mathrm{GHz}$. The source wave, $\mathbf{B}_{\mathbf{0}}$, corresponds to the $\mathrm{TE}_{1}$ mode. Domains 1 and 2 are vacuum. Analytical expressions for electric and magnetic fields being known, the relative discretization error in $\mathrm{L}_{2}$-norm, defined by

$$
\frac{1}{\max \left|E_{y \text { analytical }}\right|}\left[\int_{0}^{a}\left|E_{y}(x)-E_{y \text { analytical }}(x)\right|^{2} d x\right]^{1 / 2}
$$

is provided in Fig. 3 for the E-field, where mesh step is the edge length.

The $\mathrm{TE}_{1}$ mode expression is given by

$$
\begin{aligned}
\mathrm{B}_{0}^{\mathrm{TE}}(\mathrm{x})=\frac{\sqrt{\mathrm{Z}_{0}}}{\mathrm{Z}_{1,1}^{\mathrm{TE}}+\mathrm{Z}_{0}} \mathrm{f}_{1}(\mathrm{x}) \text { where } \mathrm{Z}_{1,1}^{\mathrm{TE}} & =\frac{j \omega \mu_{0}}{\sqrt{\left(\frac{\pi}{a}\right)^{2}-k_{0}^{2}}} \\
\text { and where } \mathrm{f}_{1}(\mathrm{x}) & =\sqrt{\frac{2}{a} \sin \left(\frac{\pi}{a} x\right) .}
\end{aligned}
$$




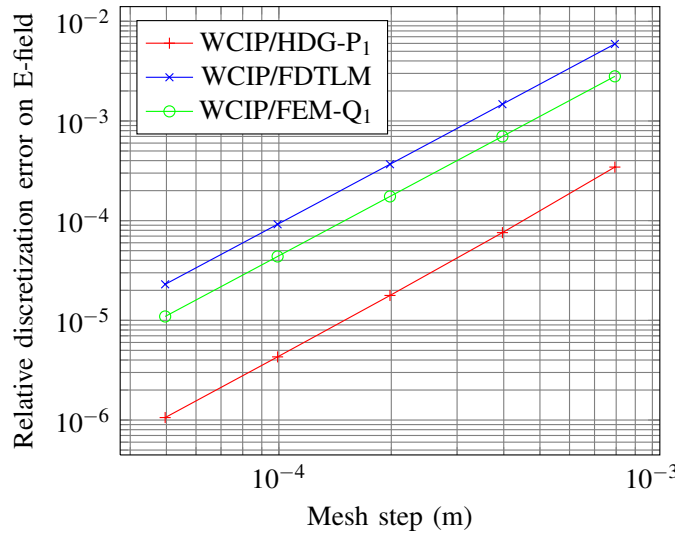

Fig. 3. Vacuum: relative discretization error in $\mathrm{L}_{2}$-norm for E-field $\left(E_{y}\right.$ component) with $\mathrm{TE}_{1}$ in excitation.

The FEM is implemented with quadrangular elements and first order approximation (FEM-Q ${ }_{1}$ ) and HDG with triangular elements and first order approximation (HDG- $\left.\mathrm{P}_{1}\right)$. The HDG$\mathrm{P}_{1}$ method provides better results as far as relative error is concerned (see Fig. 3). It is noteworthy that the HDG discretization results in more degrees of freedom. Convergence order is defined by

$$
\text { order }=\frac{\log \left(E r r e l \_h_{1}\right)-\log \left(\text { Errel_ } h_{2}\right)}{\log \left(h_{1}\right)-\log \left(h_{2}\right)}
$$

where $E r r e l \_h_{1}$ and Errel_ $h_{2}$ correspond to the relative discretisation errors in $\mathrm{L}_{2}$-norm respectively for mesh step $h_{1}$ and for refined mesh step $h_{2}$. Here, the convergence order of the three methods is 2 , which means that they converge in $h^{2}, h$ denoting the mesh step. A comparison between hybrid methods using HDG- $\mathrm{P}_{0}$, HDG- $\mathrm{P}_{1}$ and HDG- $\mathrm{P}_{2}$ [3] in domain 2 was also performed for the E-field. This comparison shows that convergence order is 1 with HDG- $\mathrm{P}_{0}, 2$ with HDG- $\mathrm{P}_{1}$ and also 2 with $\mathrm{HDG}-\mathrm{P}_{2}$ because WCIP limits convergence order, but relative error on this example is improved with $\mathrm{HDG}-\mathrm{P}_{2}$.

\section{B. Diffraction of a guided mode on a microstrip line}

A microstrip line is inserted on the surface $\Sigma$ (see Fig. 2b). It is centered and the metal proportion compared to air is $50 \%$. Since domains 1 and 2 are vacuum, dielectric permittivities are $\varepsilon_{\mathrm{r}, 1}=1$ and $\varepsilon_{\mathrm{r}, 2}=1$. In this case, an analytical solution is not available and therefore, the chosen reference is the solution obtained with the WCIP alone, meshing the domain with $N=2^{15}$ where $N$ is the number of segments on $\Sigma$. We inject the $\mathrm{TE}_{1}$ mode on the microstrip line and we calculate the relative error on the E-field and the J-current compared to the WCIP reference. Relative errors on electric field and current are respectively summarized in Tables I- IV. Mesh size represents the edge length ratio of the rectangles compared to the initial mesh. For instance, 1/2 means that the step size is twice smaller than the initial step size in both axes. Initial mesh is characterized by a step size of $794 \mu \mathrm{m}$ in both directions $x$ and $z$.

We observe that convergence orders are respectively 1 and 0.5 for E-field and J-current (order reduction coming from
TABLE I

MICROSTRIP LINE: RELATIVE DISCRETIZATION ERROR IN L2-NORM ON E-FIELD WITH WCIP/HDG.

\begin{tabular}{ccccc}
\hline Mesh size & \multicolumn{2}{c}{ WCIP/HDG-P } & \multicolumn{2}{c}{ WCIP/HDG-P 1} \\
\hline 1 & $3.09 \times 10^{-2}$ & - & $2.91 \times 10^{-2}$ & - \\
$1 / 2$ & $1.72 \times 10^{-2}$ & 0.8479 & $1.58 \times 10^{-2}$ & 0.8827 \\
$1 / 4$ & $9.08 \times 10^{-3}$ & 0.9188 & $8.23 \times 10^{-3}$ & 0.9373 \\
$1 / 8$ & $4.76 \times 10^{-3}$ & 0.9314 & $4.31 \times 10^{-3}$ & 0.9350 \\
$1 / 16$ & $2.46 \times 10^{-3}$ & 0.9517 & $2.23 \times 10^{-3}$ & 0.9507 \\
\hline
\end{tabular}

TABLE II

MICROSTRIP LINE: RELATIVE DISCRETIZATION ERROR IN L $\mathrm{L}_{2}$-NORM ON E-FIELD WITH WCIP/FDTLM AND WCIP/FEM.

\begin{tabular}{ccccc}
\hline Mesh size & \multicolumn{2}{c}{ WCIP/FDTLM } & \multicolumn{2}{c}{ WCIP/FEM-Q 1} \\
\hline 1 & $2.65 \times 10^{-2}$ & - & $2.34 \times 10^{-2}$ & - \\
$1 / 2$ & $1.47 \times 10^{-2}$ & 0.8557 & $1.34 \times 10^{-2}$ & 0.8025 \\
$1 / 4$ & $7.72 \times 10^{-3}$ & 0.9237 & $7.24 \times 10^{-3}$ & 0.8933 \\
$1 / 8$ & $4.06 \times 10^{-3}$ & 0.9262 & $3.86 \times 10^{-3}$ & 0.9059 \\
$1 / 16$ & $2.11 \times 10^{-3}$ & 0.9440 & $2.03 \times 10^{-3}$ & 0.9282 \\
\hline
\end{tabular}

TABLE III

MICROSTRIP LINE: RELATIVE DISCRETIZATION ERROR IN $\mathrm{L}_{2}$-NORM ON J-CURRENT WITH WCIP/HDG.

\begin{tabular}{ccccc}
\hline Mesh size & \multicolumn{2}{c}{ WCIP/HDG-P } & \multicolumn{2}{c}{ WCIP/HDG-P $_{1}$} \\
\hline 1 & $2.74 \times 10^{-2}$ & - & $2.76 \times 10^{-2}$ & - \\
$1 / 2$ & $1.92 \times 10^{-2}$ & 0.5114 & $1.95 \times 10^{-2}$ & 0.5048 \\
$1 / 4$ & $1.34 \times 10^{-2}$ & 0.5173 & $1.37 \times 10^{-2}$ & 0.5080 \\
$1 / 8$ & $9.34 \times 10^{-3}$ & 0.5254 & $9.59 \times 10^{-3}$ & 0.5121 \\
$1 / 16$ & $6.44 \times 10^{-3}$ & 0.5373 & $6.70 \times 10^{-3}$ & 0.5174 \\
\hline
\end{tabular}

TABLE IV

MICROSTRIP LINE: RELATIVE DISCRETIZATION ERROR IN $\mathrm{L}_{2}$-NORM ON J-CURRENT WITH WCIP/FDTLM AND WCIP/FEM.

\begin{tabular}{ccccc}
\hline Mesh size & \multicolumn{2}{c}{ WCIP/FDTLM } & \multicolumn{2}{c}{ WCIP/FEM-Q 1} \\
\hline 1 & $2.76 \times 10^{-2}$ & - & $2.77 \times 10^{-2}$ & - \\
$1 / 2$ & $1.95 \times 10^{-2}$ & 0.5044 & $1.95 \times 10^{-2}$ & 0.5037 \\
$1 / 4$ & $1.37 \times 10^{-2}$ & 0.5073 & $1.37 \times 10^{-2}$ & 0.5064 \\
$1 / 8$ & $9.61 \times 10^{-3}$ & 0.5111 & $9.65 \times 10^{-3}$ & 0.5097 \\
$1 / 16$ & $6.72 \times 10^{-3}$ & 0.5160 & $6.76 \times 10^{-3}$ & 0.5139 \\
\hline
\end{tabular}

the discontinuity between metal and dielectric) in TE case whatever method used in domain 2 , with very close relative discretization errors between hybrid methods. Furthermore, convergence orders are the same between hybrid methods WCIP/HDG- $\mathrm{P}_{0}$ and WCIP/HDG- $\mathrm{P}_{1}$, probably because of the low regularity of the solution. These results motivate the polynomial adaptivity when there are metal and dielectric discontinuities at the interface.

\section{Diffraction of a guided mode on a microstrip line printed} on an inhomogeneous substrate

On Fig. 2b, a microstrip line is printed on a substrate of permittivity $\varepsilon_{\mathrm{r}, 2}$, surrounded by two layers of permittivity $\varepsilon_{\mathrm{r}, 1}$. We take here $\varepsilon_{\mathrm{r}, 2}=5$ and $\varepsilon_{\mathrm{r}, 1}=1$, i.e. vacuum. Our reference is obtained meshing hybrid method WCIP/FEM-Q ${ }_{1}$ with $N=$ $2^{10}$. Relative discretization errors are provided in Table $\mathrm{V}$ and VI. For the E-field, convergence order is close to 1 and for the J-current, it is between 0.5 and 1 .

In order to observe the influence of GMRES on the number of iterations needed to solve the linear system (4), a compari- 
TABLE V

MICROSTRIP LINE ON INHOMOGENEOUS SUBSTRATE: RELATIVE DISCRETIZATION ERROR IN L 2 -NORM ON E-FIELD.

\begin{tabular}{ccccc}
\hline Mesh size & \multicolumn{2}{c}{ WCIP/FEM-Q 1} & \multicolumn{2}{c}{ WCIP/FDTLM } \\
\hline & Relative error & Order & Relative error & Order \\
\hline 1 & $2.22 \times 10^{-2}$ & - & $2.48 \times 10^{-2}$ & - \\
$1 / 2$ & $1.25 \times 10^{-2}$ & 0.8308 & $1.34 \times 10^{-2}$ & 0.8824 \\
$1 / 4$ & $6.50 \times 10^{-3}$ & 0.9388 & $6.87 \times 10^{-3}$ & 0.9689 \\
$1 / 8$ & $3.26 \times 10^{-3}$ & 0.9959 & $3.40 \times 10^{-3}$ & 1.0145 \\
$1 / 16$ & $1.50 \times 10^{-3}$ & 1.1164 & $1.56 \times 10^{-3}$ & 1.1222 \\
\hline
\end{tabular}

TABLE VI

MICROSTRIP LINE ON INHOMOGENEOUS SUBSTRATE: RELATIVE DISCRETIZATION ERROR IN L2-NORM ON J-CURRENT.

\begin{tabular}{ccccc}
\hline Mesh size & \multicolumn{2}{c}{ WCIP/FEM-Q 1} & \multicolumn{2}{c}{ WCIP/FDTLM } \\
\hline & Relative error & Order & Relative error & Order \\
\hline 1 & $2.11 \times 10^{-2}$ & - & $2.06 \times 10^{-2}$ & - \\
$1 / 2$ & $1.43 \times 10^{-2}$ & 0.5615 & $1.39 \times 10^{-2}$ & 0.5725 \\
$1 / 4$ & $9.37 \times 10^{-3}$ & 0.6126 & $8.97 \times 10^{-3}$ & 0.6296 \\
$1 / 8$ & $5.82 \times 10^{-3}$ & 0.6879 & $5.47 \times 10^{-3}$ & 0.7126 \\
$1 / 16$ & $3.31 \times 10^{-3}$ & 0.8137 & $3.07 \times 10^{-3}$ & 0.8366 \\
\hline
\end{tabular}

TABLE VII

ITERATION NUMBER ACCORDING TO SOLVER.

\begin{tabular}{ccccc}
\hline Mesh size & \multicolumn{2}{c}{ WCIP/FEM-Q 1} & \multicolumn{2}{c}{ WCIP/FDTLM } \\
\hline & GMRES & BICGSTAB & GMRES & BICGSTAB \\
\hline 1 & 12 & 12 & 12 & 12 \\
$1 / 2$ & 27 & 30 & 25 & 26 \\
$1 / 4$ & 48 & 51 & 46 & 49 \\
$1 / 8$ & 66 & 80 & 63 & 90 \\
$1 / 16$ & 84 & 147 & 82 & 128 \\
\hline
\end{tabular}

son with another iterative method (BICGSTAB [8]) is achieved in Table VII. We can conclude that GMRES requires less iterations, in particular for fine meshes.

\section{Diffraction of a guided mode on a perfect sheet with WCIP 2D/HDG $3 D$}

We present preliminary results which correspond to the test case already considered in subsection III-A but now in 3D. Here TE and TM modes are coupled. Furthermore, the FMT is quite different from the 2D case but the linear system (4) is unchanged. Geometry of domain 2 is therefore a cube of side $1.27 \mathrm{~cm}$ meshed in a structured way with tetrahedra in domain $2 . \Sigma$ is a surface meshed with rectangles that are called pixels. The connection between meshes is achieved taking $\mathbf{A}_{2}$ values at the center of the pixels on $z=0$. Each $\mathbf{A}_{\mathbf{2}}$ value is consequently imposed on the hypotenuse of two neighboring triangles. We calculate in Table VIII relative discretization error on surface on E-field when a $\mathrm{TE}_{10}$ mode is injected at $f_{0}=16 \mathrm{GHz}$. As in $2 \mathrm{D}$ case, analytical solution is available and it is the reference. Only non-zero tangential component is dealt with, i.e. $\mathrm{E}_{\mathrm{y}}$. Initial mesh is characterized by 48 tetrahedra.

Convergence order for the E-field is between 2 en 3 when surface relative discretization error is calculated.

\section{CONCLUSION}

In this work, we have realized a feasibility study of the hybridization technique between the WCIP and other volumic
TABLE VIII

SURFACE RELATIVE DISCRETIZATION ERROR IN $\mathrm{L}_{2}$-NORM ON E-FIELD

\begin{tabular}{ccc}
\hline Mesh size & \multicolumn{2}{c}{$\mathrm{E}_{\mathrm{y}}$} \\
\hline & Relative error & Order \\
\hline 1 & $1.15 \times 10^{-2}$ & - \\
$1 / 2$ & $1.59 \times 10^{-3}$ & 2.8502 \\
$1 / 4$ & $2.23 \times 10^{-4}$ & 2.8346 \\
$1 / 8$ & $3.62 \times 10^{-5}$ & 2.6260 \\
\hline
\end{tabular}

methods. A convergence order of 2 has been emphasized in a canonical case whatever the hybrid method (FEM-Q ${ }_{1}, \mathrm{HDG}$ or FDTLM) and using HDG- ${ }_{2}$ does not improve convergence order. The insertion of a microstrip line between both domains is also relevant, because the 3 methods provide similar results, namely a convergence order of 1 for E-field and an order of 0.5 for electric current for a $\mathrm{TE}_{1}$ mode in excitation. We manage to tackle the intrinsic inhomogeneity problem of the WCIP comparing hybrid methods WCIP/FEM-Q ${ }_{1}$ and WCIP/FDTLM. The test case was a microstrip line printed on an inhomogeneous substrate. Convergence orders of 1 for E-field and between 0.5 and 1 for J-current were found. It constitutes the basis for 3D work. Consequently, we implemented the same procedure for the hybridization between WCIP-2D and HDG-3D, with a preliminary validation considering the vacuum case. It yielded a convergence order is between 2 and 3, which is in accordance with expectations from 2D results. These results are rather promising for considering more complex 3D cases, namely calculations on electric currents on planar circuits enforcing electronic specific functions.

\section{ACKNOWLEDGMENT}

The authors would like to thank the Defence Procurement Agency (DGA) which supports the first author.

\section{REFERENCES}

[1] S. Wane, D. Bajon, H. Baudrand and P. Gammand, "A new full-wave hybrid differential-integral approach for the investigation of multilayer structures including nonuniformly doped diffusions", IEEE MTT., vol. 53, no. 1, pp. 200-213, January 2005.

[2] M. Titaouine, N. Raveu and H. Baudrand, "Wave Concept Iterative Procedure for inhomogeneous multi-layered circuits", in Circuits and Systems and TAISA Conference, 2009. NEWCAS-TAISA '09. Joint IEEE North-East Workshop on , pp. 1-3, 2009.

[3] L. Li, S. Lanteri and R. Perrussel, "Numerical investigation of a high order hybridizable discontinuous Galerkin method for $2 \mathrm{~d}$ time-harmonic Maxwell's equations", COMPEL, vol. 2, no. 3, pp. 1112-1138, 2013.

[4] A. Zugari, M. Khalladi, M. Iben Yaich, N. Raveu and H. Baudrand, "New approach: WCIP and FDTLM hybridization", in Microwave Symposium (MMS), 2009 Mediterrannean , pp. 1-4, 2009.

[5] C. Girard, N. Raveu, R. Perrussel, S. Lanteri, "Hybridization between the WCIP and volumic methods", "Hybridation entre la WCIP et des méthodes volumiques", in Journées Nationales Microondes (JNM), May 2013 (in French).

[6] C. Girard, N. Raveu, S. Lanteri and R. Perrussel, "1d WCIP and FEM hybridization", in Numelec, 2012.

[7] Y. Saad and M.H. Schultz, "GMRES: A Generalized Minimal Residual Algorithm for solving non symmetric linear systems ", in SIAM J.Sci.and Stat. Comput., vol. 7, no. 3, July 1986.

[8] H.A. van der Vorst, "Bi-CGSTAB: A Fast and Smoothly Converging Variant of Bi-CG for the Solution of Nonsymmetric Linear Systems ", in SIAM J.Sci.and Stat. Comput., vol. 13, no. 2, pp. 631-644, March 1992. 